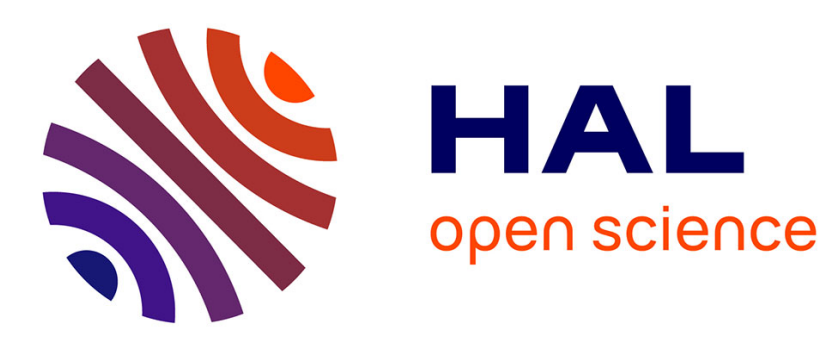

\title{
Consistent Discretization of Locally Homogeneous Finite-time Stable Control Systems
}

Andrey Polyakov, Denis Efimov, Bernard Brogliato, Markus Reichhartinger

\section{To cite this version:}

Andrey Polyakov, Denis Efimov, Bernard Brogliato, Markus Reichhartinger. Consistent Discretization of Locally Homogeneous Finite-time Stable Control Systems. ECC 2019 - 18th European Control Conference, Jun 2019, Naples, Italy. pp.1616-1621, 10.23919/ECC.2019.8795633 . hal-02069717

\section{HAL Id: hal-02069717 https://hal.inria.fr/hal-02069717}

Submitted on 15 Mar 2019

HAL is a multi-disciplinary open access archive for the deposit and dissemination of scientific research documents, whether they are published or not. The documents may come from teaching and research institutions in France or abroad, or from public or private research centers.
L'archive ouverte pluridisciplinaire HAL, est destinée au dépôt et à la diffusion de documents scientifiques de niveau recherche, publiés ou non, émanant des établissements d'enseignement et de recherche français ou étrangers, des laboratoires publics ou privés. 


\title{
Consistent Discretization of Locally Homogeneous Finite-time Stable Control Systems
}

\author{
Andrey Polyakov ${ }^{1}$, Denis Efimov ${ }^{1}$, Bernard Brogliato ${ }^{2}$ and Markus Reichhartinger ${ }^{3}$
}

\begin{abstract}
An algorithm of consistent implicit discretization for locally homogeneous finite-time stable system having discontinuity only at the origin is developed. It preserves finite-time stability property in the discrete-time models. The homogeneous domination approach is utilized for analysis of the discretized model. The scheme is demonstrated for a simplified model of a quadrotor control system.
\end{abstract}

\section{INTRODUCTION}

Discretization issues are important for a digital implementation of estimation and control algorithms as well as for a computer simulation of control processes. Construction of a so-called consistent stable discretization is a non-trivial problem for essentially non-linear ordinary differential equations (ODEs), which do not satisfy some classical regularity assumptions. In our case consistent discretization means that the resulting discrete-time model approximates solutions and preserves stability/convergence properties of the original continuous-time system. An inconsistent discretization of non-Lipschitz feedback algorithms results in degradation of control precision [1], chattering effect [2], or even instability [3]. For example, the sliding mode algorithms are known to be difficult in practical realization [1], [4], [5] due to their discontinuous (set-valued) nature, which may involve chattering caused by the discretization (the so-called numerical chattering). The mentioned papers have discovered that the implicit discretization technique is useful for practical implementation of non-smooth and discontinuous control and estimation algorithms. In particular, numerical chattering suppression in both input and output, as well as a good closed-loop performance have been confirmed experimentally in [1], [6].

A symmetry of functions or vector fields with respect to a certain group of transformations (dilations) is known as homogeneity [7], [8], [9], [10]. Nonlinear homogeneous differential equations/inclusions form an important class of control systems [11], [12], [13], [14], [15]. They can be utilized as local approximations [8], [14] or set-valued extensions [16], [17] of nonlinear control systems. For example, some models of process control [18], nonholonomic mechanical systems [19] and systems with frictions [16] are homogeneous or at least locally homogeneous. It is well known that any

\footnotetext{
${ }^{1}$ Andrey Polyakov and Denis Efimov are with Inria Lille, Univ. Lille, CNRS, UMR 9189 - CRIStAL, (F-59000 Lille, France) and with ITMO University, 49 av. Kronverskiy, Saint-Petersburg, 197101, Russia

${ }^{2}$ Bernard Brogliato is with Univ. Grenoble Alpes, Inria, CNRS, Grenoble INP, LJK, Grenoble, 38000, France bernard.brogliatodinria.fr

${ }^{3}$ Markus Reichhartinger is with Graz University of Technology, Austria
}

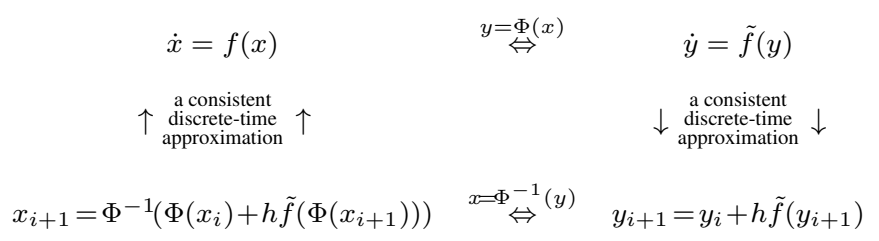

Fig. 1. The scheme of the consistent implicit discretization of $\dot{x}=f(x)$

asymptotically stable homogeneous system with a negative degree is finite-time stable

The type of homogeneity is identified by the dilation group, which can be linear [7], [20], [21] or nonlinear [9], [11]. In this paper, we deal with the linear geometric homogeneity studied originally in [10] for infinite dimensional models. In the finite dimensional case the corresponding dilation group (see [21] for more details) is given by the matrix exponential function $e^{s G_{\mathrm{d}}}$, where $s \in \mathbb{R}$ is the group parameter and $G_{\mathbf{d}} \in \mathbb{R}^{n \times n}$ is an anti-Hurwitz matrix known also as a generator of the dilation.

Recently [22], a problem of consistent discretization of homogeneous finite-dimensional systems with negative degree has been studied. In particular, a discretization algorithm preserving the finite-time stability in the obtained discretetime model has been developed. It is essentially based on topological equivalence of any stable homogeneous system to a quadratically stable one (see Fig. 1 and [22], [23]).

It is shown [24] that an implementation of finite-time controllers using the developed scheme rejects numerical chattering and essentially improves the control precision. This paper continues this research direction and proposes a consistent discretization scheme for non-homogeneous finitetime stable systems, which admit homogeneous approximation at the origin.

Notation $\|A\|=\sup _{\|x\|=1}\|A x\|$ and $\lfloor A\rfloor=\inf _{\|x\|=1}\|A x\|$ for $A \in \mathbb{R}^{n \times n} ; I_{n} \in \mathbb{R}^{n \times n}$ is the identity matrix; 0 denotes zero element; $\operatorname{diag}\left\{\lambda_{1}, . ., \lambda_{n}\right\}$ - diagonal matrix with elements $\lambda_{i}$; $P \succ 0$ means positive definiteness of $P=P^{\top} \in \mathbb{R}^{n \times n}$; $\lambda_{\max }(P)$ and $\lambda_{\min }(P)$ denote maximal and minimal eigenvalues of $P=P^{\top} \in \mathbb{R}^{n \times n} ; \Re(\lambda)$ denotes the real part of a complex number $\lambda$; the notation $P^{\frac{1}{2}}$ means that $P^{\frac{1}{2}}=M$ is such that $P=M^{2}$; the class $\mathcal{K}$ consists of continuous strictly increasing functions $\sigma:[0,+\infty) \rightarrow[0,+\infty), \sigma(0)=0$; $B(r)=\left\{x \in \mathbb{R}^{n}:\|x\|<r\right\}$ is an open ball of the radius $r>0$; the closed ball is denoted by $\bar{B}(r)$; a mapping $\mathbb{R}^{n} \rightrightarrows \mathbb{R}^{m}$ is set-valued if it associates a set of $\mathbb{R}^{m}$ with each $x \in \mathbb{R}^{n}$. 


\section{Problem Statement}

Let us consider the non-linear system

$$
\dot{x}=f(x), \quad t>0, \quad x(0)=x_{0},
$$

where $x(t) \in \mathbb{R}^{n}$ is the system state and the nonlinear function $f: \mathbb{R}^{n} \rightarrow \mathbb{R}^{n}$ is continuous on $\mathbb{R}^{n} \backslash\{\mathbf{0}\}$, i.e., the only possible discontinuity point of $f$ is the origin. System (1) is assumed to be forward complete for solutions understood in the sense of Filippov [25]:

An absolutely continuous function $\phi\left(\cdot, x_{0}\right):[0,+\infty) \rightarrow$ $\mathbb{R}^{n}$ is a solution to (1) if $\phi\left(0, x_{0}\right)=x_{0}$ and for almost all $t>0$ it satisfies the differential inclusion

$$
\dot{x} \in F(x)=\bigcap_{\varepsilon>0} \bigcap_{\mu(N)=0} \operatorname{cof}(x+B(\varepsilon) \backslash\{N\}),
$$

where $B(\varepsilon)$ denotes the ball in $\mathbb{R}^{n}$ of the radius $\varepsilon$, co denotes convex closure and $\mu(N)$ means that the Lebesgue measure of the set $N \subset \mathbb{R}^{n}$ is zero. In our case, $F(x)=\{f(x)\}$ is a singleton for $x \in \mathbb{R}^{n} \backslash\{\mathbf{0}\}$, but

$$
F(\mathbf{0})=\bigcap_{\varepsilon>0} \operatorname{cof}(B(\varepsilon) \backslash\{\mathbf{0}\})
$$

is a set, each time $f$ is discontinuous at $\mathbf{0}$.

Recall [16], [26] that the origin of system (1) is said to be locally uniformly finite-time stable, if it is Lyapunov stable, and there exist a neighborhood $U$ of the origin and a locally bounded function $T: U \rightarrow[0,+\infty)$ such that any solution $\phi\left(\cdot, x_{0}\right)$ to (1) satisfies $\phi\left(t, x_{0}\right)=0$ for $t \geq T\left(x_{0}\right), x_{0} \in U$.

We understand the consistency of a discretization scheme for the finite-time stable system (1) in the sense of the following definition.

Definition 1: [24] A (possibly) set-valued mapping

$$
Q: \mathbb{R}_{+} \times \mathbb{R}^{n} \times \mathbb{R}^{n} \rightrightarrows \mathbb{R}^{n}
$$

is said to be a consistent discrete-time approximation of the locally uniformly finite-time stable system (1) in a neighborhood $U$ of the origin if $\exists h_{0}>0$ :

- Existence property: for any $\tilde{x} \in U$ and any $h \in\left(0, h_{0}\right)$, there exists $\tilde{x}_{h} \in U$ :

$$
\mathbf{0} \in Q\left(h, \tilde{x}, \tilde{x}_{h}\right),
$$

and $\tilde{x}_{h}=\mathbf{0}$ is the unique solution to $\mathbf{0} \in Q\left(h, \mathbf{0}, \tilde{x}_{h}\right)$.

- Finite-time convergence property: for any $h \in\left(0, h_{0}\right)$ each sequence $\left\{x_{i}\right\}_{i=0}^{+\infty}$ generated by the inclusion

$$
\mathbf{0} \in Q\left(h, x_{i}, x_{i+1}\right), \quad i=0,1,2, \ldots .
$$

converges to zero in a finite number of steps, i.e., $\forall x_{0} \in$ $U \backslash\{\mathbf{0}\} i^{*}>0$ such that $x_{i}=\mathbf{0}$ for $i \geq i^{*}$.

- Approximation property: for any $\varepsilon>0$ and any $R>\varepsilon$, there exists a function $\omega \in \mathcal{K}$ such that any sequence $\left\{x_{i}\right\}_{i=0}^{+\infty}$ generated by (4) satisfies

$$
\left\|\phi\left(h, x_{i}\right)-x_{i+1}\right\| \leq h \omega(h),
$$

provided that $x_{i+1}, x_{i} \in U$ and $\left\|x_{i+1}\right\|,\left\|x_{i}\right\| \in[\varepsilon, R]$, where $\phi\left(\cdot, x_{i}\right)$ is a solution to (1) with the initial condition $x(0)=x_{i}$.
Notice that the last property in this definition requires the existence of the conventional estimate (5) for the discretization error on any compact set from $U \backslash\{\mathbf{0}\}$ (since $\varepsilon>0$ and $R>\varepsilon$ can be selected arbitrary small and arbitrary large, respectively). The origin is excluded because of a possible singularity of the vector field $f$ at zero (in particular, it can be discontinuous at the origin). The inequality (5) describes a local (one-step) approximation error. An approximation error on the time interval $\left[0, T\left(x_{0}\right)\right]$ is $O(\omega(h))$ provided that $h=T\left(x_{0}\right) / N$ with $N \in \mathbb{N}$. This error tends to zero as $h \rightarrow 0$, i.e., as $N \rightarrow+\infty$.

The aim of the paper is to design a consistent (in the sense of Definition 1) discretization scheme for locally finite-time stable system (1), under the assumption that the vector field $f$ admits a local homogeneous approximation at the origin (see [14] or the next section for the details).

\section{Preliminaries}

\section{A. Generalized Homogeneity}

The generalized homogeneity [10], [23], [21], [27] deals with the group of linear transformations (linear dilations).

Definition 2: A map $\boldsymbol{d}: \mathbb{R} \rightarrow \mathbb{R}^{n \times n}$ is called dilation in $\mathbb{R}^{n}$ if it satisfies

- Group property: $\boldsymbol{d}(0)=I_{n}$ and $\boldsymbol{d}(t+s)=\boldsymbol{d}(t) \boldsymbol{d}(s)=$ $\boldsymbol{d}(s) \boldsymbol{d}(t)$ for all $t, s \in \mathbb{R}$;

- Continuity property: $d$ is a continuous map, i.e.,

$\forall t \in \mathbb{R}, \forall \varepsilon>0, \exists \delta>0:|s-t|<\delta \Rightarrow\|\boldsymbol{d}(s)-\boldsymbol{d}(t)\| \leq \varepsilon ;$

- Limit property: $\lim _{s \rightarrow-\infty}\|\boldsymbol{d}(s) x\|=0$ and $\lim _{s \rightarrow+\infty}\|\boldsymbol{d}(s) x\|=$ $+\infty$ uniformly on the unit sphere $S:=\{x:\|x\|=1\}$.

The dilation $\mathbf{d}$ is a continuous group of invertible linear maps $\mathbf{d}(s) \in \mathbb{R}^{n \times n}, \mathbf{d}(-s)=[\mathbf{d}(s)]^{-1}$. The matrix $G_{\mathbf{d}} \in \mathbb{R}^{n \times n}$ $G_{\mathbf{d}}=\lim _{s \rightarrow 0} \frac{\mathbf{d}(s)-I}{s}$ is known [28, Chapter 1] as the generator of the group $\mathbf{d}$. It satisfies the following properties $\frac{d \mathbf{d}(s)}{d s}=G_{\mathbf{d}} \mathbf{d}(s), \mathbf{d}(s)=e^{G_{\mathbf{d}} s}=\sum_{i=0}^{+\infty} \frac{s^{i} G_{\mathbf{d}}^{i}}{i !}, s \in \mathbb{R}$. The most popular dilations in $\mathbb{R}^{n}$ are [17], [16] the uniform (or standard) dilation (L. Euler 17 th century) : $\mathbf{d}_{1}(s)=$ $e^{s} I_{n}, \quad s \in \mathbb{R}$; and the weighted dilation (Zubov 1958, [7]): $\mathbf{d}_{2}(s)=\operatorname{diag}\left\{e^{r_{i} s}\right\}, \quad s \in \mathbb{R}, r_{i}>0, i=1, \ldots, n$. They satisfy Definition 2 with $G_{\mathbf{d}_{1}}=I_{n}$ and $G_{\mathbf{d}_{2}}=\operatorname{diag}\left\{r_{i}\right\}$, respectively. In fact, any anti-Hurwitz ${ }^{1}$ matrix $G_{\mathbf{d}} \in \mathbb{R}^{n \times n}$ defines a dilation $\mathbf{d}(s)=e^{G_{\mathbf{d}} s}$ in $\mathbb{R}^{n}$.

Definition 3: [21] The dilation $\boldsymbol{d}$ is strictly monotone if there exists $\beta>0$ such that $\|\boldsymbol{d}(s)\| \leq e^{\beta s}$ as $s<0$.

Obviously, the monotonicity of a dilation may depend on the norm $\|\cdot\|$ in $\mathbb{R}^{n}$.

Theorem 1: [21] Let $\boldsymbol{d}$ be a dilation in $\mathbb{R}^{n}$, then

1) all eigenvalues $\lambda_{i}$ of the matrix $G_{\boldsymbol{d}}$ are placed in the right complex half-plane, i.e., $\Re\left(\lambda_{i}\right)>0, i=1,2, \ldots, n$;

2) there exists a matrix $P \in \mathbb{R}^{n \times n}$ such that

$$
P G_{\boldsymbol{d}}+G_{\boldsymbol{d}}^{\top} P \succ 0, \quad P=P^{\top} \succ 0 ;
$$

\footnotetext{
${ }^{1}$ The matrix $G_{\mathbf{d}} \in \mathbb{R}^{n}$ is anti-Hurwitz if $-G_{\mathbf{d}}$ is Hurwitz.
} 
3) the dilation $\boldsymbol{d}$ is strictly monotone with respect to the weighted Euclidean norm $\|\cdot\|=\sqrt{\langle\cdot, \cdot\rangle}$ induced by the inner product $\langle x, z\rangle=x^{\top} P z$ with $P$ satisfying (6).

Moreover,

$$
\begin{aligned}
& e^{\alpha s} \leq\lfloor\boldsymbol{d}(s)\rfloor \leq\|\boldsymbol{d}(s)\| \leq e^{\beta s} \quad \text { if } \quad s \leq 0, \\
& e^{\beta s} \leq\lfloor\boldsymbol{d}(s)\rfloor \leq\|\boldsymbol{d}(s)\| \leq e^{\alpha s} \quad \text { if } \quad s \geq 0,
\end{aligned}
$$

where $\alpha=\frac{1}{2} \lambda_{\max }\left(P^{\frac{1}{2}} G_{\boldsymbol{d}} P^{-\frac{1}{2}}+P^{-\frac{1}{2}} G_{\boldsymbol{d}}^{\top} P^{\frac{1}{2}}\right)$ and $\beta=$ $\frac{1}{2} \lambda_{\min }\left(P^{\frac{1}{2}} G_{\boldsymbol{d}} P^{-\frac{1}{2}}+P^{-\frac{1}{2}} G_{\boldsymbol{d}}^{\top} P^{\frac{1}{2}}\right)$.

The latter theorem proves that any dilation $\mathbf{d}$ is strictly monotone if $\mathbb{R}^{n}$ is equipped with the norm $\|x\|=\sqrt{x^{\top} P x}$, provided that the matrix $P \succ 0$ satisfies (6).

Definition 4: [21] A continuous function $p: \mathbb{R}^{n} \rightarrow \mathbb{R}_{+}$is said to be a d-homogeneous norm if $p(x) \rightarrow 0$ as $x \rightarrow \mathbf{0}$ and $p(\boldsymbol{d}(s) x)=e^{s} p(x)>0$ for $x \in \mathbb{R}^{n} \backslash\{\mathbf{0}\}$ and $s \in \mathbb{R}$.

Obviously, the $\mathbf{d}$-homogeneous norm is neither a norm nor semi-norm in the general case, since the triangle inequality may not hold. However, many authors (see e.g. [14], [27] and references therein) call a function satisfying the latter definition a homogeneous norm. We follow this tradition.

The canonical homogeneous norm $\|\cdot\|_{\boldsymbol{d}}: \mathbb{R}^{n} \rightarrow \mathbb{R}_{+}$is defined as

$$
\|x\|_{\boldsymbol{d}}=e^{s_{x}} \text { where } s_{x} \in \mathbb{R}:\left\|\boldsymbol{d}\left(-s_{x}\right) x\right\|=1 .
$$

The map $\|\cdot\|_{\mathbf{d}}: \mathbb{R}^{n} \rightarrow[0,+\infty)$ is well defined and single-valued for monotone dilations [27]. In [29] such a homogeneous norm was called canonical because it is induced by a (canonical) norm in $\mathbb{R}^{n}$. Notice that

$$
\left\lfloor\mathbf{d}\left(\ln \|x\|_{\mathbf{d}}\right)\right\rfloor \leq\|x\| \leq\left\|\mathbf{d}\left(\ln \|x\|_{\mathbf{d}}\right)\right\| \quad \text { for } \quad x \in \mathbb{R}^{n},
$$

and, due to (7), $\|\cdot\|_{\mathbf{d}}$ is continuous at zero.

Proposition 1: [21] If $\boldsymbol{d}$ is a strictly monotone dilation then

- the canonical homogeneous norm $\|\cdot\|_{\boldsymbol{d}}$ is Lipschitz continuous on $\mathbb{R}^{n} \backslash\{\mathbf{0}\}$;

- if the norm $\|\cdot\|$ is smooth outside the origin then the homogeneous norm $\|\cdot\|_{d}$ is also smooth outside the origin, $\frac{d\|\boldsymbol{d}(-s) x\|}{d s}<0$ if $s \in \mathbb{R}, x \in \mathbb{R}^{n} \backslash\{\mathbf{0}\}$ and

$$
\frac{\partial\|x\|_{d}}{\partial x}=\left.\frac{\left.\|x\|_{d} \frac{\partial\|z\|}{\partial z}\right|_{z=d(-s) x}}{\left.\frac{\partial\|z\|}{\partial z}\right|_{z=d(-s) x} G_{d} d(-s) x}\right|_{s=\ln \|x\|_{d}}
$$

Below we use the notation $\|\cdot\|_{d}$ only for the canonical homogeneous norm induced by the weighted Euclidean norm $\|x\|=\sqrt{x^{\top} P x}$ with a matrix $P \succ 0$ satisfying (6). The unit sphere $S$ is defined using the same norm.

Vector fields, which are homogeneous with respect to dilation d, have many properties useful for control design and state estimation of linear and nonlinear plants as well as for analysis of convergence rates [20].

Definition 5: [21] A vector field $f: \mathbb{R}^{n} \rightarrow \mathbb{R}^{n}$ (resp. a function $h: \mathbb{R}^{n} \rightarrow \mathbb{R}$ ) is said to be $\boldsymbol{d}$-homogeneous if there exists $\nu \in \mathbb{R}$

$$
\begin{aligned}
& f(\mathbf{d}(s) x)=e^{\nu s} \mathbf{d}(s) f(x), \forall x \in \mathbb{R}^{n} \backslash\{\mathbf{0}\}, \forall s \in \mathbb{R} . \\
& \left(\text { resp. } h(\mathbf{d}(s) x)=e^{\nu s} h(x), \forall x \in \mathbb{R}^{n} \backslash\{\mathbf{0}\}, \forall s \in \mathbb{R} .\right)
\end{aligned}
$$

The number $\nu \in \mathbb{R}$ is called the homogeneity degree of $f$ (resp. $h$ ).
Let $\mathbb{F}_{\boldsymbol{d}}\left(\mathbb{R}^{n}\right)$ (resp. $\mathbb{H}_{\boldsymbol{d}}\left(\mathbb{R}^{n}\right)$ ) be the set of vector fields $\mathbb{R}^{n} \rightarrow \mathbb{R}^{n}$ (resp. functions $\mathbb{R}^{n} \rightarrow \mathbb{R}$ ) satisfying the identity (10) (resp. (11)), which are continuous on $\mathbb{R}^{n} \backslash\{\mathbf{0}\}$. Let $\operatorname{deg}_{\mathbb{F}_{d}}(f)$ (resp. $\operatorname{deg}_{\mathbb{H}_{d}}(h)$ ) denote the homogeneity degree of $f \in \mathbb{F}_{\boldsymbol{d}}\left(\mathbb{R}^{n}\right)\left(\right.$ resp. $h \in \mathbb{H}_{\boldsymbol{d}}\left(\mathbb{R}^{n}\right)$ ).

The homogeneity allows local properties (e.g., smoothness) of vector fields (functions) to be extended globally [7], [8]. For instance [21], the vector field $f \in \mathbb{F}_{\mathbf{d}}\left(\mathbb{R}^{n}\right)$ is Lipschitz continuous on $\mathbb{R}^{n} \backslash\{\boldsymbol{0}\}$ if and only if it satisfies Lipschitz condition on the unit sphere $S$. Similarly, since the map $s \rightarrow \mathbf{d}(s) x$ is locally uniformly continuous, then uniform continuity of $f \in \mathbb{F}_{\mathbf{d}}\left(\mathbb{R}^{n}\right)$ on the unit sphere implies its local uniform continuity on $\mathbb{R}^{n} \backslash\{\mathbf{0}\}$ (see [24]).

\section{B. Quadratic Stability of Nonlinear Homogeneous Systems}

Homogeneity may simplify the analysis of differential equations. The most important property of $\mathbf{d}$-homogeneous systems is the symmetry of solutions [7], [11], [12],[30], [20]. Namely, if $\varphi_{\xi_{0}}:[0, T) \rightarrow \mathbb{R}^{n}$ is a solution to

$$
\dot{\xi}=f(\xi), \quad f \in \mathbb{F}_{\boldsymbol{d}}\left(\mathbb{R}^{n}\right)
$$

with the initial condition $\xi(0)=\xi_{0} \in \mathbb{R}^{n}$, then $\varphi_{\boldsymbol{d}(s) \xi_{0}}:\left[0, e^{-\nu s} T\right) \rightarrow \mathbb{R}^{n}$ defined as $\varphi_{\boldsymbol{d}(s) \xi_{0}}(t)=$ $\boldsymbol{d}(s) \varphi_{\xi_{0}}\left(t e^{\nu s}\right), \quad s \in \mathbb{R}$ is a solution to (12) with the initial condition $\xi(0)=\boldsymbol{d}(s) \xi_{0}$, where $\nu=\operatorname{deg}_{\mathbb{F}_{\boldsymbol{d}}}(f)$.

The latter property implies many corollaries. In this paper we use the next one.

Theorem 2: [21] The following five claims are equivalent:

1) The origin of the system (12) is asymptotically stable.

2) There exists a Lyapunov function $V$ for the system (12) such that $V \in \mathbb{H}_{\boldsymbol{d}}\left(\mathbb{R}^{n}\right) \cap C^{\infty}\left(\mathbb{R}^{n}\right)$.

3) The origin of the system

$$
\dot{z}=\|z\|^{1+\operatorname{deg}_{\mathbb{F}_{d}}(f)}\left(\frac{\left(I_{n}-G_{d}\right) z z^{\top} P}{z^{\top} P G_{d} z}+I_{n}\right) f\left(\frac{z}{\|z\|}\right)
$$

is asymptotically stable, where $z=\sqrt{z^{\top} P z}$, and the positive definite matrix $P \in \mathbb{R}^{n \times n}$ satisfies (6).

4) For any positive definite matrix $P \in \mathbb{R}^{n \times n}$ satisfying (6) there exists

$$
\Psi \in \mathbb{F}_{\boldsymbol{d}}\left(\mathbb{R}^{n}\right) \cap C^{\infty}\left(\mathbb{R}^{n} \backslash\{\mathbf{0}\}\right), \quad \operatorname{deg}_{\mathbb{F}_{\boldsymbol{d}}}(\Psi)=0,
$$

such that $\Psi$ is a diffeomorphism on $\mathbb{R}^{n} \backslash\{\mathbf{0}\}$, a homeomorphism on $\mathbb{R}^{n}, \Psi(\mathbf{0})=\mathbf{0}$ and

$$
\frac{\partial\left(\Psi^{\top}(\xi) P \Psi(\xi)\right)}{\partial \xi} f(\xi)<0 \quad \text { if } \Psi^{\top}(\xi) P \Psi(\xi)=1 .
$$

Moreover, $\|\Psi\|_{\boldsymbol{d}} \in \mathbb{H}_{\boldsymbol{d}}\left(\mathbb{R}^{n}\right) \cap C^{\infty}\left(\mathbb{R}^{n} \backslash\{\mathbf{0}\}\right)$ is a Lyapunov function for the system (12).

5) For any matrix $P \in \mathbb{R}^{n \times n}$ satisfying (6), there exists a smooth mapping $\Xi \in C^{\infty}\left(\mathbb{R}^{n} \backslash\{\mathbf{0}\}, \mathbb{R}^{n \times n}\right)$,

$$
\operatorname{det}(\Xi(z)) \neq 0, \quad \frac{\partial \Xi(z)}{\partial z_{i}} z=0, \quad \Xi\left(e^{s} z\right)=\Xi(z)
$$$$
\text { for } z=\left(z_{1}, \ldots, z_{n}\right)^{\top} \in \mathbb{R}^{n} \backslash\{\mathbf{0}\}, s \in \mathbb{R}, i=1, \ldots, n
$$

$z^{\top} \Xi^{\top}(z) P \Xi(z)\left(\frac{\left(I_{n}-G_{d}\right) z z^{\top} P}{z^{\top} P G_{d} z}+I_{n}\right) f\left(\frac{z}{\|z\|}\right)<0$.

This theorem proves two important facts:

- Any generalized homogeneous system (12) is homeomorphic on $\mathbb{R}^{n}$ and diffeomorphic on $\mathbb{R}^{n} \backslash\{\mathbf{0}\}$ to a 
standard homogeneous one (13). The corresponding change of coordinates is given by

$$
z=\Phi(\xi):=\|\xi\|_{\mathbf{d}} \mathbf{d}\left(-\ln \|\xi\|_{\mathbf{d}}\right) \xi
$$

and $\xi=\Phi^{-1}(z):=\mathbf{d}(\ln \|z\|) \frac{z}{\|z\|}$.

- Any asymptotically stable generalized homogeneous system is homeomorphic on $\mathbb{R}^{n}$ and diffeomorphic on $\mathbb{R}^{n} \backslash\{\mathbf{0}\}$ to a quadratically stable one. Indeed, making the change of variables $z=\Psi(\xi)$ we derive

$$
\dot{z}=\hat{f}(z)=\left.\frac{\partial \Psi(\xi)}{\partial \xi} f(\xi)\right|_{\xi=\Psi^{-1}(z)},
$$

but the criterion (14) implies that $z^{\top} P \dot{z}<0$ if $z^{\top} P z=$ 1 , so the homogeneous norm $\|\cdot\|_{\mathbf{d}}$ is the Lyapunov function to the latter system. Finally, the change of variable $x=\|z\|_{\mathbf{d}} \mathbf{d}\left(-\ln \|z\|_{\mathbf{d}}\right) z$ gives $\|z\|_{\mathbf{d}}=\|x\|$, so the transformed system $\dot{x}=\tilde{f}(x)$ is quadratically stable with the Lyapunov function $V$ defined as $V(x)=$ $\|x\|^{2}=x^{\top} P x$, where

$$
\tilde{f}(x)=\|x\|^{1+\operatorname{deg}_{\mathbb{F}_{\mathbf{d}}}(f)}\left(\frac{\left(I_{n}-G_{\mathbf{d}}\right) x x^{\top} P}{x^{\top} P G_{\mathbf{d}} x}+I_{n}\right) \hat{f}\left(\frac{x}{\|x\|}\right) .
$$

Below we show that the transformations $\Phi$ and $\Psi$ can be utilized for the design of a consistent discretization scheme for locally homogeneous systems.

Recall [16], [17], [20], [21] that if the homogeneous system (12) is asymptotically stable and $\operatorname{deg}_{\mathbb{F}_{\mathbf{d}}}(f)<0$, then it is globally uniformly finite-time stable.

Remark 1: If $\boldsymbol{d}$ is a dilation with the generator $G_{\boldsymbol{d}}$, then for any fixed $\alpha>0$, the group $\boldsymbol{d}^{\alpha}$ defined as $\boldsymbol{d}^{\alpha}(s):=$ $\boldsymbol{d}(\alpha s), s \in \mathbb{R}^{n}$, is the dilation with the generator $G_{\boldsymbol{d}^{\alpha}}=$ $\alpha G_{\boldsymbol{d}}$. If $f \in \mathbb{F}_{\boldsymbol{d}}\left(\mathbb{R}^{n}\right)$, then $f \in \mathbb{F}_{\boldsymbol{d}^{\alpha}}\left(\mathbb{R}^{n}\right)$ and $\operatorname{deg}_{\mathbb{F}_{\boldsymbol{d}^{\alpha}}}(f)=$ $\alpha \operatorname{deg}_{\mathbb{F}_{d}}(f)$. In other words, if $\operatorname{deg}_{\mathbb{F}_{d}}(f)<0$, then a new dilation $\boldsymbol{d}^{\alpha}$ can be selected such that $\operatorname{deg}_{\mathbb{F}_{\boldsymbol{d}^{\alpha}}}(f)=-1$.

\section{Local Homogeneity}

Local homogeneous approximations has been studied in [14], [31] in the case of for weighted dilations. In the generalized case local homogeneity is defined analogously.

Definition 6: A vector field $f_{0} \in \mathbb{F}_{\boldsymbol{d}}\left(\mathbb{R}^{n}\right)$ is said to be a dhomogeneous approximation of a vector field $f: \mathbb{R}^{n} \rightarrow \mathbb{R}^{n}$ at 0 if $\lim _{s \rightarrow-\infty}\left\|e^{-\operatorname{deg}_{\mathbb{F}_{\boldsymbol{d}}}\left(f_{0}\right) s} \boldsymbol{d}(-s) f(\boldsymbol{d}(s) x)-f_{0}(x)\right\|=0$ uniformly on $x \in S$.

Local homogeneity at $\infty$ can be introduced similarly considering the limit $s \rightarrow+\infty$.

The next simple result follows the well-known ideas of homogeneous domination approach [7], [14].

Lemma 1: Let $f_{0} \in \mathbb{F}_{\boldsymbol{d}}\left(\mathbb{R}^{n}\right)$ be a local d-homogeneous approximation of a vector field $f: \mathbb{R}^{n} \rightarrow \mathbb{R}^{n}$ at $\mathbf{0}$ and $\operatorname{deg}_{\mathbb{F} \boldsymbol{d}}\left(f_{0}\right)<0$. If the system

$$
\dot{x}=f_{0}(x)
$$

is asymptotically stable then the system (1) is locally finitetime stable.

A consistent discretization scheme for finite-time stable homogeneous systems is developed [24] based on Theorem 2. Below we use similar ideas in order to design a consistent discretization scheme locally (close to 0 ), where the dhomogeneous approximation $f_{0}$ is a dominating nonlinearity. The next trivial corollary plays a key role in the later constructions.

Corollary 1: If all conditions of Lemma 1 hold, then for any matrix $P \in \mathbb{R}^{n \times n}$ satisfying (6), there exist $r>0$ and a smooth mapping $\Xi \in C^{\infty}\left(\mathbb{R}^{n} \backslash\{\mathbf{0}\}, \mathbb{R}^{n \times n}\right), \operatorname{det}(\Xi(z)) \neq$ $0, \quad \frac{\partial \Xi(z)}{\partial z_{i}} z=0, \quad \Xi\left(e^{s} z\right)=\Xi(z)$, for $z=\left(z_{1}, \ldots, z_{n}\right)^{\top} \in$ $\mathbb{R}^{n} \backslash\{\mathbf{0}\}, s \in \mathbb{R}, i=1, \ldots, n$, such that the inequality

$z^{\top} \Xi^{\top}(z) P \Xi(z)\left(\frac{\left(I_{n}-G_{\boldsymbol{d}}\right) z z^{\top} P}{z^{\top} P G_{\boldsymbol{d}} z}+I_{n}\right) \boldsymbol{d}(-\ln \|z\|) f\left(\frac{\boldsymbol{d}(\ln \|z\|) z}{\|z\|}\right)<0$

holds for any $z \in B(r) \backslash\{\mathbf{0}\}$.

\section{Finite-Time Stable Implicit Discretization}

The main idea of the design of a finite-time stable discretization for homogeneous systems, is to use the coordinate transformation (16).

Theorem 3: Let a vector field $f: \mathbb{R}^{n} \rightarrow \mathbb{R}^{n}$ be locally uniformly continuous on any ring

$$
\begin{gathered}
K\left(r_{1}, r_{2}\right)=\left\{x \in \mathbb{R}^{n}: r_{1} \leq\|x\| \leq r_{2}\right\}, \\
0<r_{1}<r_{2}<r^{\max }<+\infty
\end{gathered}
$$

and admit a d-homogeneous approximation $f_{0} \in \mathbb{F}_{\boldsymbol{d}}\left(\mathbb{R}^{n}\right)$ at 0 such that $\operatorname{deg}_{\mathbb{F}_{d}}\left(f_{0}\right)=-1$ and the condition (15) with $\Xi=I_{n}$ holds for $f_{0}$.

Then there exists $r>0$ such that the map $Q: \mathbb{R}_{+} \times \mathbb{R}^{n} \times$ $\mathbb{R}^{n} \rightrightarrows \mathbb{R}^{n}$ defined as

$$
\begin{gathered}
Q\left(h, x_{i}, x_{i+1}\right)=\tilde{Q}\left(h, \Phi\left(x_{i}\right), \Phi\left(x_{i+1}\right)\right), \\
\Phi(x)=\|x\|_{\boldsymbol{d}} \boldsymbol{d}\left(-\ln \|x\|_{\boldsymbol{d}}\right) x, \\
\tilde{Q}\left(h, y_{i}, y_{i+1}\right)=y_{i+1}-y_{i}-h \tilde{F}\left(y_{i+1}\right), \\
\tilde{F}(y)=\bigcap_{\varepsilon>0} \operatorname{cof}(y+B(\varepsilon) \backslash\{\mathbf{0}\}),
\end{gathered}
$$

$\tilde{f}(y):=\|y\|\left(\frac{\left(I-G_{\boldsymbol{d}}\right) y y^{\top} P}{y^{\top} G_{\boldsymbol{d}} P y}+I_{n}\right) \boldsymbol{d}(-\ln \|y\|) f\left(\boldsymbol{d}(\ln \|y\|) \frac{y}{\|y\|}\right)$, is a consistent discrete-time approximation in

$$
U=\left\{x \in \mathbb{R}^{n}:\|x\|_{\boldsymbol{d}} \leq r / 2\right\}
$$

of the locally uniformly finite-time stable system (1) with the maximum step size $h_{0}=\frac{r}{2 \sup _{y \in \mathbb{R}^{n}:\|y\| \leq r} \tilde{f}(y)}$.

The latter theorem is based on the fact that the system $\dot{y}=\tilde{f}_{0}(y)$ admits a quadratic Lyapunov function (the condition (15) with $\Xi=$ const). However, as it was shown in Theorem 2, any stable homogeneous system is equivalent to a quadratically stable one. If $f$ in Theorem 3 is replaced with the equivalent one:

$$
f^{n e w}(x)=\left.\frac{\partial \Psi(\xi)}{\partial \xi} f(\xi)\right|_{\xi=\Psi^{-1}(x)}, \quad x \in \mathbb{R}^{n}, \quad \xi \in \mathbb{R}^{n},
$$

where $\Psi \in \mathbb{F}_{\mathbf{d}}\left(\mathbb{R}^{n}\right)$ is a diffeomorphism on $\mathbb{R}^{n} \backslash\{\mathbf{0}\}$ given in Theorem 2, then the condition $\Xi=I_{n}$ required for Theorem 3 is fulfilled.

According to Theorem 2, a homogeneous Lyapunov function $V \in \mathbb{H}_{\mathbf{d}}\left(\mathbb{R}^{n}\right) \cap C^{\infty}\left(\mathbb{R}^{n}\right)$ with degree $\operatorname{deg}_{\mathbb{H}}(V)=\mu>0$ 
can always be found for any asymptotically stable system with a homogeneous vector field $f \in \mathbb{F}_{\mathbf{d}}\left(\mathbb{R}^{n}\right)$. In this case, the required transformation $\Psi$ can be defined as follows

$$
\Psi(\xi)=\mathbf{d}\left(\ln \frac{V^{1 / \mu}(\xi)}{\|\xi\|_{\mathbf{d}}}\right) \xi .
$$

(see [23] for more details).

In other words, if we know a homogeneous Lyapunov function for a finite-time stable homogeneous system then, we can easily design a consistent (in the sense of Definition 1) implicit discretization scheme.

Therefore, any finite-time stable system allowing a finitetime stable homogeneous approximation at $\mathbf{0}$ admits a consistent implicit approximation, at least locally.

\section{ExAmple: Discrete-time Approximation of A QUADROTOR CONTROL SYSTEM}

Let us consider the system

$\dot{x}=A(x) x+B u, \quad A(x)=\left(\begin{array}{cccc}0 & I & 0 & 0 \\ 0 & 0 & E(x) & 0 \\ 0 & 0 & 0 & I \\ 0 & 0 & 0 & 0\end{array}\right), \quad B=\left(\begin{array}{l}0 \\ 0 \\ 0 \\ I\end{array}\right)$,

where $x \in \mathbb{R}^{8}, u \in \mathbb{R}^{2}, E(x)=\left(\begin{array}{cc}\frac{\sin \left(x_{5}\right)}{x_{5}} & 0 \\ 0 & \frac{\sin \left(x_{6}\right) \cos \left(x_{5}\right)}{x_{6}}\end{array}\right), I=\left(\begin{array}{ll}1 & 0 \\ 0 & 1\end{array}\right)$.

The system of this form appears as a simplified model for the control of horizontal position of a quadrotor, when the vertical position and a yaw angle are already stabilized in some set-points.

Obviously, $A(x) x$ admits d-homogeneous approximation $A_{0} x$ at $\mathbf{0}$ with the homogeneity degree $-1, \mathbf{d}(s)=e^{s G_{\mathbf{d}}}$ and

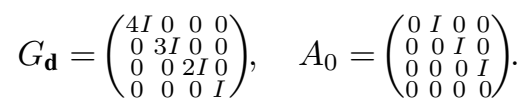

Let the control law is defined as

$$
u(x)=K \mathbf{d}\left(-\ln \|x\|_{\mathbf{d}}\right) x
$$

where $K \in \mathbb{R}^{2 \times 8}$ such that

$$
\begin{gathered}
\left(A_{0}+B K\right)^{\top} P+P\left(A_{0}+B K\right)+\left(G_{\mathbf{d}} P+P G_{\mathbf{d}}\right)=0, \\
G_{\mathbf{d}} P+P G_{\mathbf{d}} \succ 0, \quad P \succ 0 .
\end{gathered}
$$

Such a selection of $K$ is always possible (see [32]). Moreover, it can be shown that the control $u$ has globally bounded and discontinuity only at the origin. The closed-loop system is locally finite-time stable, $V=\|x\|_{\mathbf{d}}$ is a Lyapunov function and $f_{0}(x)=A_{0} x+B u(x)$ is a d-homogeneous approximation of the closed-loop system at zero, $\operatorname{deg}_{\mathbb{F}_{\mathbf{d}}}\left(f_{0}\right)=-1$. All conditions of Theorem 3 are fulfilled. Applying the coordinate transformation $y=\Phi(x)=\|x\|_{\mathbf{d}} \mathbf{d}\left(-\ln \|x\|_{\mathbf{d}}\right) x$ we derive

$$
\begin{aligned}
& \tilde{f}(y)=\left(A_{0}+B K+\left(1-\frac{y^{\top} P \Delta A(y) y}{y^{\top} G_{\mathbf{d}} P y}\right)\left(G_{\mathbf{d}}-I\right)+\Delta A(y)\right) \frac{y}{\|y\|}, \\
& \Delta A=\left(\begin{array}{cccc}
0 & 0 & 0 & 0 \\
0 & 0 & E-I & 0 \\
0 & 0 & 0 & 0 \\
0 & 0 & 0 & 0
\end{array}\right), E=\left(\begin{array}{cc}
\frac{\sin \left(\|y\| y_{5}\right)}{\|y\| y_{5}} & 0 \\
0 & \frac{\sin \left(\|y\| y_{6}\right) \cos \left(\|y\| y_{5}\right)}{\|y\| y_{6}}
\end{array}\right) .
\end{aligned}
$$

Obviously, $\tilde{F}(y)=\tilde{f}(y)$ if $y \neq 0$ and

$$
\tilde{F}(0)=\left(A_{0}+B K+G_{\mathbf{d}}-I\right) B(1) \subset \mathbb{R}^{n} .
$$

Hence, if $y_{i} \in h \tilde{F}(0)$ then the inclusion $y_{i+1} \in y_{i}+$ $h \tilde{F}\left(y_{i+1}\right)$ has a solution $y_{i+1}=0$. For the case $y_{i} \neq$ $h \tilde{F}\left(y_{i+1}\right)$ some properly adapted numerical procedures (like Newton method) is required for solving of the obtained discrete-time inclusion $y_{i} \in y_{i+1}-h \tilde{F}\left(y_{i+1}\right)$. Notice, that $\tilde{f}$ is smooth outside the origin, so the Newton method can be applied while, for example, $y_{i+1}^{[k]} \notin h \tilde{F}(0)$, where $k$ denotes the number of Newton's iteration. The latter inclusion can be utilized as one of conditions for termination of the Newton iterations, since next the state of the system converges to zero in one step.

Let us denote $q_{i+1}=\left\|y_{i+1}\right\|, z_{i+1}=\frac{y_{i+1}}{\left\|y_{i+1}\right\|}$ and $\tilde{A}=$ $A_{0}+B K+G_{\mathbf{d}}$. Similarly to [24] the discretized control can be defined as $u_{i}=u\left(x_{i+1}\right)=u\left(\Phi^{-1}(y)\right)=K z_{i+1}$. If $y_{i}^{\top}\left(I_{n}-\tilde{A}\right)^{-\top} P\left(I_{n}-\tilde{A}\right)^{-1} y_{i} \leq h^{2}$ then $y_{i} \in h \tilde{F}(0)$,

$$
q_{i+1}=0 \quad \text { and } \quad z_{i+1}=h^{-1}\left(I_{n}-\tilde{A}\right)^{-1} y_{i}
$$

Otherwise, according the scheme proposed above the value of $y_{i+1}$ (as well as $q_{i+1} \neq 0$ and $z_{i+1}$ ) are assumed to be derived by means of a Newton method. Notice that the matrix $I-\tilde{A}$ is invertible [24], so the control $u_{i}$ is well defined. The original state is given by $x_{i+1}=\mathbf{d}\left(\ln \left\|y_{i+1}\right\|\right) y_{i+1} /\left\|y_{i+1}\right\|$.

The simulation results for the consistent discretization scheme with $h=0.04$ are given in Fig. 2 and the results for the explicit Euler discretization with the same sampling period are depicted in Fig. 3. They confirm finite-time convergence of the consistent discretization scheme and the numerical chattering of the explicit one.
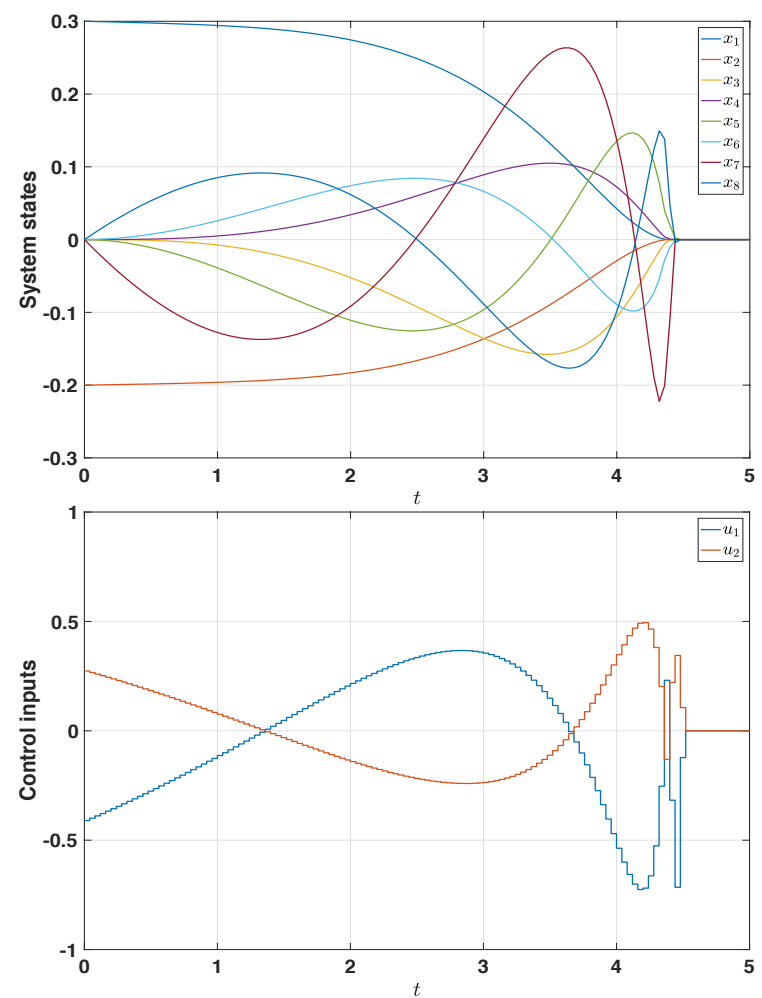

Fig. 2. Simulation results for the consistently discretized model. 

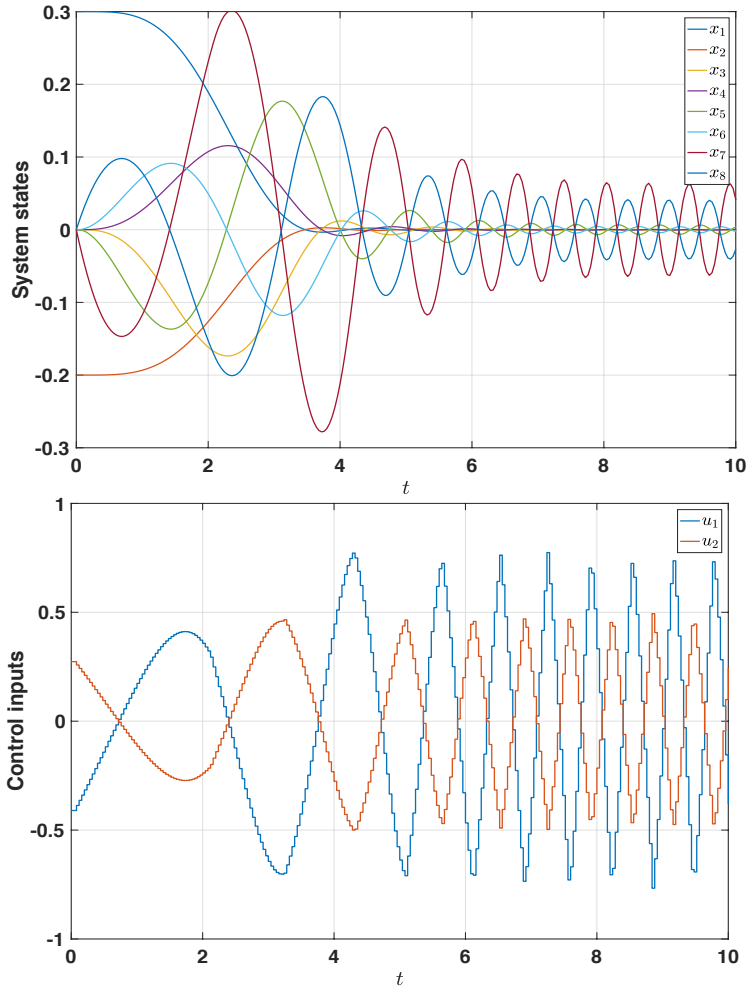

Fig. 3. Simulation results for the explicit Euler method.

\section{Discussions AND CONCLUSIONS}

In the paper it is shown that any homogeneous finitetime stable system allowing finite-time stable homogeneous approximation at $\mathbf{0}$ always admits an implicit discretization scheme preserving finite-time convergence. For practical implementation, implicit discretization scheme needs to be supported with an appropriated algorithm of solving the corresponding implicit equation/inclusion (18). Development of such algorithms is planned as future research.

\section{ACKNOWLEDGMENTS}

The authors thank the support of the French National Research Agency (ANR), Project ANR-18-CE40-0008 "DIGITSLID". The first and the second authors also acknowledge the support of the Government of Russian Federation (Grant 08-08) and the Ministry of Education and Science of Russian Federation (Project 14.Z50.31.0031).

\section{REFERENCES}

[1] O. Huber, V. Acary, and B. Brogliato, "Lyapunov stability and performance analysis of the implicit discrete sliding mode control." IEEE Transactions on Automatic Control, vol. 61, no. 10, pp. 3016-3030, 2016.

[2] V. I. Utkin, Sliding Modes in Control Optimization. Berlin: SpringerVerlag, 1992.

[3] A. Levant, "On fixed and finite time stability in sliding mode control," in IEEE Conference on Decision and Control, 2013, pp. 4260-4265.

[4] V. Acary, B. Brogliato, and Y. Orlov, "Chattering-free digital slidingmode control with state observer and disturbance rejection," IEEE Transactions on Automatic Control, vol. 57, no. 5, pp. 1087-1101, 2012.

[5] R. Kikuuwe, S. Yasukouchi, H. Fujimoto, and M. Yamamoto, "Proxybased sliding mode control: A safer extension of pid position control," IEEE Transactions on Robotics, vol. 26, no. 4, pp. 670-683, 20110.

[6] O. Huber, B. Brogliato, V. Acary, A. Boubakir, F. Plestan, and B. Wang, Recent Trends in Sliding Mode Control. IET, London, 2016, ch. Experimental results on implicit and explicit time-discretization of equivalent control-based sliding mode control, pp. 207-235.
[7] V. Zubov, "On systems of ordinary differential equations with generalized homogenous right-hand sides," Izvestia vuzov. Mathematica (in Russian)., vol. 1, pp. 80-88, 1958.

[8] H. Hermes, "Nilpotent approximations of control systems and distributions," SIAM Journal of Control and Optimization, vol. 24, no. 4, p. $731,1986$.

[9] L. Rosier, "Etude de quelques problèmes de stabilization," PhD Thesis, Ecole Normale Superieure de Cachan (France), 1993.

[10] A. Polyakov, D. Efimov, E. Fridman, and W. Perruquetti, "On homogeneous distributed parameters equations," IEEE Transactions on Automatic Control, vol. 61, no. 11, pp. 3657-3662, 2016.

[11] M. Kawski, "Geometric homogeneity and stabilization," in Proc. IFAC Nonlinear Control Symposium, A. Krener and D. Mayne, Eds., Lake Tahoe, CA, 1995, pp. 164-169.

[12] L. Rosier, "Homogeneous Lyapunov function for homogeneous continuous vector field," Systems \& Control Letters, vol. 19, pp. 467-473, 1992.

[13] W. Perruquetti, T. Floquet, and E. Moulay, "Finite-time observers: application to secure communication," IEEE Transactions on Automatic Control, vol. 53, no. 1, pp. 356-360, 2008.

[14] V. Andrieu, L. Praly, and A. Astolfi, "Homogeneous Approximation, Recursive Observer Design, and Output Feedback," SIAM Journal of Control and Optimization, vol. 47, no. 4, pp. 1814-1850, 2008.

[15] A. Polyakov, D. Efimov, and W. Perruquetti, "Finite-time and fixedtime stabilization: Implicit Lyapunov function approach," Automatica, vol. 51, no. 1, pp. 332-340, 2015.

[16] Y. Orlov, "Finite time stability and robust control synthesis of uncertain switched systems," SIAM Journal of Control and Optimization, vol. 43, no. 4, pp. 1253-1271, 2005.

[17] A. Levant, "Homogeneity approach to high-order sliding mode design," Automatica, vol. 41, no. 5, pp. 823-830, 2005.

[18] K. Zimenko, D. Efimov, A. Polyakov, and W. Perruquetti, "A note on delay robustness for homogeneous systems with negative degree," Automatica, vol. 79, no. 5, pp. 178-184, 2017.

[19] J.-B. Pomet and C. Samson, "Time-varying exponential stabilization of nonholonomic systems in power form,” Inria, RR-2126, 1993.

[20] S. P. Bhat and D. S. Bernstein, "Geometric homogeneity with applications to finite-time stability," Mathematics of Control, Signals and Systems, vol. 17, pp. 101-127, 2005.

[21] A. Polyakov, "Sliding mode control design using canonical homogeneous norm," International Journal of Robust and Nonlinear Control, vol. 29 , no. 3, pp. 682-701, 2018.

[22] A. Polyakov, D. Efimov, and B. Brogliato, "Consistent discretization of finite-time and fixed-time stable systems," SIAM Journal of Control and Optimization, vol. 57, no. 1, pp. 78-103, 2019.

[23] A. Polyakov, "Quadratic-like stabilizability of homogeneous systems," in Proc. Conference on Decision and Control, Melbourne, Australia, 2017, pp. 577-582.

[24] A. Polyakov, D. Efimov, and B. Brogliato, "Consistent discretization of finite-time stable homogeneous systems," in 15th International Workshop on Variable Structure Systems and Sliding Mode Control, 2018.

[25] A. F. Filippov, Differential Equations with Discontinuous Right-hand Sides. Kluwer Academic Publishers, 1988.

[26] A. Polyakov and L. Fridman, "Stability notions and Lyapunov functions for sliding mode control systems," Journal of The Franklin Institute, vol. 351, no. 4, pp. 1831-1865, 2014.

[27] A. Polyakov, J.-M. Coron, and L. Rosier, "On homogeneous finitetime control for linear evolution equation in hilbert space," IEEE Transactions on Automatic Control, vol. 63, no. 9, pp. 3143-3150, 2018.

[28] A. Pazy, Semigroups of Linear Operators and Applications to Partial Differential Equations. Springer, 1983.

[29] A. Polyakov, J.-M. Coron, and L. Rosier, "On finite-time stabilization of evolution equations: A homogeneous approach," in Conference on Decision and Control, 2016, pp. 3143-3148.

[30] L. Grune, "Homogeneous state feedback stabilization of homogeneous systems," SIAM Journal of Control and Optimization, vol. 38, no. 4, pp. 1288-1308, 2000.

[31] D. Efimov and W. Perruquetti, "Oscillations conditions in homogenous systems," in Proc. IFAC NOLCOS Symp., 2010, pp. 1379-1384.

[32] A. Polyakov, D. Efimov, and W. Perruquetti, "Robust stabilization of MIMO systems in finite/fixed time," International Journal of Robust and Nonlinear Control, vol. 26, no. 1, pp. 69-90, 2016. 